Article

\title{
On the Uniqueness Results and Value Distribution of Meromorphic Mappings
}

\author{
Rahman Ullah ${ }^{1}$, Xiao-Min Li ${ }^{1}$, Faiz Faizullah ${ }^{2, *}$, Hong-Xun $\mathrm{Yi}^{3}$ and Riaz Ahmad Khan ${ }^{4}$ \\ 1 School of Mathematical Sciences, Ocean University of China, Qingdao 266100, China; \\ rahman_yzi@yahoo.com (R.U.); xmli01267@gmail.com (X.-M.L.) \\ 2 Department of BS \& H, College of E \& ME, National University of Sciences and Technology (NUST), \\ Islamabad 44000, Pakistan \\ 3 Department of Mathematics, Shandong University, Jinan 250100, China; hxyi@sdu.edu.cn \\ 4 SMME, National University of Sciences and Technology (NUST), Islamabad 44000, Pakistan; \\ riaz.ahmad@smme.nust.edu.pk \\ * Correspondence: faiz_math@ceme.nust.edu.pk; Tel.: +92-333-987-8193
}

Academic Editor: Lei Ni

Received: 9 June 2017; Accepted: 10 August 2017; Published: 17 August 2017

\begin{abstract}
This research concentrates on the analysis of meromorphic mappings. We derived several important results for value distribution of specific difference polynomials of meromorphic mappings, which generalize the work of Laine and Yang. In addition, we proved uniqueness theorems of meromorphic mappings. The difference polynomials of these functions have the same fixed points or share a nonzero value. This extends the research work of Qi, Yang and Liu, where they used the finite ordered meromorphic mappings.
\end{abstract}

Keywords: meromorphic mappings; value distribution; difference polynomials; uniqueness results

\section{Introduction}

Let $\Omega$ be the set of finite linear measure of positive real numbers, which may not be the same at every occurrence. Assume $T(q, \alpha)$ denotes the Nevanlinna characteristic of a nonconstant meromorphic mapping $\alpha$ and $S(q, \alpha)$ represents any quantity fulfilling $S(q, \alpha)=o\{T(q, \alpha)\}$, as $q \rightarrow \infty$ and $q \notin \Omega$. Consider a point $c$ in the extended plane. Indicate two nonconstant meromorphic mappings by $\alpha$ and $\beta$. The mappings $\alpha$ and $\beta$ share the value $c \mathrm{IM}$, if they have the same $c$-points ignoring multiplicities [1]. Also, $c$ is called a small mapping of $\alpha$, provided that $c$ is a meromorphic mapping fulfilling $T(q, c)=S(q, \alpha)$ [1]. All through the current paper, we consider meromorphic mappings in the complex plane and represent the order of $\alpha$ by $\rho(\alpha)$. Consider the following result which was proved by Clunie [2] and Hayman [3]:

Theorem 1. Suppose $k \geq 1$ be a positive integer. Let $\alpha(y)$ represents a transcendental entire mapping. Then there are infinitely many zeros of $\alpha^{k}(y) \alpha^{\prime}(y)-1$.

Reading Theorem 1, the following problem arises:

Problem 1. Let $\lambda \neq 0$ be a complex number. What will be the conclusion of Theorem 1 if $\alpha^{k}(y) \alpha^{\prime}(y)$ of Theorem 1 is replaced with $\alpha^{k}(y) \alpha(y+\lambda)$ or $\alpha^{k}(y) \Delta_{\lambda} \alpha(y)$ for a transcendental meromorphic mapping $\alpha(y)$ ?

In this direction, Laine and Yang [4] derived the following result to deal with Problem 1:

Theorem 2. Let $\lambda \neq 0$ be a complex number and $\alpha(y)$ be a finite order transcendental entire mapping. Then $\alpha(y)^{k} \alpha(y+\lambda)$ assumes every finite nonzero value $c$ infinitely often for $k \geq 2$. 
We now give the following two examples, for details see $[4,5]$.

Example 1. Let $\alpha(y)=1+e^{y}$. Then $\alpha(y) \alpha(y+\pi i)-1=-e^{2 y}$ has no zeros. This example shows that Theorem 2 does not remain valid if $k=1$.

Example 2. Let $\alpha(y)=e^{-e^{y}}$. Then $\alpha(y)^{2} \alpha(y+\lambda)-2=-1$ and $\rho(\alpha)=\infty$, where $\lambda$ is a nonzero constant satisfying $e^{\lambda}=-2$. Evidently, $\alpha(y)^{2} \alpha(y+\lambda)-2$ have no zeros. This example shows that Theorem 2 does not remain valid if $\alpha$ is of infinite order.

Recently Liu and Yang proved the following result [5]:

Theorem 3. Let $k \geq 2$ be an integer and $\lambda \neq 0$ be a complex number. Assume that $\alpha(y)$ be a finite order transcendental entire mapping. Let $P(y) \not \equiv 0$ be a polynomial. Then there are infinitely many zeros of $\alpha^{k}(y) \alpha(y+\lambda)-P(y)$.

We recall the following two examples from [5].

Example 3. Let $\alpha(y)=e^{-e^{y}}$. Then $\alpha(y)^{k} \alpha(y+\lambda)-P(y)=1-P(y)$ and $\rho(\alpha)=\infty$, where $\eta$ is a nonzero constant satisfying $e^{\lambda}=-k, P(y)$ is a nonconstant polynomial, $k$ is a positive integer. Evidently, $\alpha(y)^{k} \alpha(y+\lambda)-P(y)$ has finitely many zeros. This example shows that the condition " $\rho(\alpha)<\infty$ " in Theorem 3 is necessary.

In addition to Theorems 2 and 3 to deal with Problem 1 we will prove the following theorem:

Theorem 4. Let $k \geq 7$ be an integer. Suppose that the order of a transcendental meromorphic mapping $\alpha$ is given by $\rho(\alpha)=\rho<\infty$. Let $\lambda$ be a nonconstant complex number and $\Delta_{\lambda} \alpha \neq \equiv 0$. Assume $P \not \equiv 0$ be a polynomial. Then as $q \longrightarrow \infty$ and $q \notin \Omega$

$$
(k-6) T(q, \alpha) \leq \bar{N}\left(q, \frac{1}{\alpha^{k} \Delta_{\lambda} \alpha-P}\right)+o\left(\frac{T(q, \alpha)}{q^{1-\varepsilon}}\right)+O(\log q),
$$

where $\Omega \subset(1,+\infty)$ is a subset of finite logarithmic measure.

The following definition is borrowed from [6] which will be used in the forthcoming work of this article.

Definition 1. Let $\alpha$ be a nonconstant meromorphic function. We define difference operators as $\Delta_{\lambda} \alpha(y)=\alpha(y+\lambda)-\alpha(y), \Delta_{\lambda}^{n} \alpha(y)=\Delta_{\lambda}^{n-1}\left(\Delta_{\lambda} \alpha(y)\right)$, where $\lambda$ is a nonzero complex number, $n \geq 2$ is a positive integer. If $\lambda=1$, we denote $\Delta_{\lambda} \alpha(y)=\Delta \alpha(y)$. Moreover,

$$
\Delta_{\lambda}^{n} \alpha(y)=\sum_{j=0}^{n}\left(\begin{array}{l}
n \\
j
\end{array}\right)(-1)^{n-j} \alpha(y+j \lambda)
$$

The proof of Theorem 4 yields the following interesting result, which will be proved in Section 3.

Theorem 5. Let $k \geq 3$ be an integer. Suppose that the order of a transcendental entire mapping $\alpha$ is given by $\rho(\alpha)=\rho<\infty$. Let $\lambda$ be a nonconstant complex number and $\Delta_{\lambda} \alpha \neq \equiv$. Assume that $P \not \equiv 0$ be a polynomial. Then as $q \longrightarrow \infty$ and $q \notin \Omega$,

$$
(k-2) T(q, \alpha) \leq \bar{N}\left(q, \frac{1}{\alpha^{k} \Delta_{\lambda} \alpha-P}\right)+o\left(\frac{T(q, \alpha)}{q^{1-\varepsilon}}\right)+O(\log q),
$$

where $\Omega \subset(1,+\infty)$ is a subset of finite logarithmic measure. 
Now consider the example given below, which indicates that the condition " $\rho(\alpha)<\infty$ " in Theorems 4 and 5 is necessary.

Example 4. Let $\alpha(y)=e^{-e^{y}}$. Then $\rho(\alpha)=\infty$ and $\alpha(y)^{k} \Delta_{\lambda} \alpha(y)-1=-e^{(k+1) e^{y}}$ has no zeros, where $k \in Z$ and $\lambda$ is a nonzero constant satisfying $e^{\lambda}=-k$.

From Theorems 4 and 5 we can get the following results respectively.

Corollary 1. Let $k \geq 7$ be an integer. Suppose that the order of transcendental meromorphic mapping $\alpha$ is given by $\rho(\alpha)<\infty$. Consider a nonconstant complex number such that $\Delta_{\lambda} \alpha \not \equiv 0$. Assume that $P \not \equiv 0$ be a polynomial. Then there are infinitely many zeros of $\alpha^{k} \Delta_{\lambda} \alpha-P$.

Corollary 2. Let $k \geq 3$ be an integer. Suppose that the order of a transcendental entire mapping $\alpha$ is given by $\rho(\alpha)<\infty$. Let $\lambda$ be a nonconstant complex number such that $\Delta_{\lambda} \alpha \not \equiv 0$. Suppose that $P \not \equiv 0$ be a polynomial. Then there are infinitely many zeros of $\alpha^{k} \Delta_{\lambda} \alpha-P$.

Corresponding to Theorem 2, the following uniqueness theorem was derived by Qi-Yang-Liu [7].

Theorem 6. Suppose that $k \geq 6$ be an integer and $\lambda \neq 0$ be a complex number. Let the distinct transcendental entire mappings $\alpha$ and $\beta$ have finite orders. Assume that $\alpha(y)^{k} \alpha(y+\lambda)-y$ and $\beta(y)^{k} \beta(y+\lambda)-y$ share 0 CM. Then $\alpha=t \beta$, where $t \neq 1$ is a constant fulfilling $t^{k+1}=1$.

He further studied the following result [7].

Theorem 7. Let $k \geq 6$ is an integer and $\lambda \neq 0$ is a complex number. Assume that the distinct transcendental entire mappings $\alpha$ and $\beta$ have finite orders. Let $\alpha(y)^{k} \alpha(y+\lambda)$ and $\beta(y)^{k} \beta(y+\lambda)$ share 1 CM. Then $\alpha=t \beta$, where $t \neq 1$ is a constant fulfilling $t^{k+1}=1$.

From Theorem 4 we will prove the following uniqueness results for meromorphic mappings associated to difference operators.

Theorem 8. Suppose that $k \geq 12$ be an integer and $P \neq \equiv 0$ be a polynomial. Let the distinct transcendental meromorphic mappings $\alpha$ and $\beta$ have finite orders. Assume that $\lambda \neq 0$ be a complex number such that $\Delta_{\lambda} \alpha \not \equiv 0$ and $\Delta_{\lambda} \beta \not \equiv 0$. Suppose that $\alpha^{k} \Delta_{\lambda} \alpha-P$ and $\beta^{k} \Delta_{\lambda} \beta-P$ share 0 CM. Then

(i) If $k \geq 12$ and if $\alpha^{k} \Delta_{\lambda} \alpha / P$ is a Möbius transformation of $\beta^{k} \Delta_{\lambda} \beta / P$, then $\left[\alpha^{k} \Delta_{\lambda} \alpha\right]\left[\beta^{k} \Delta_{\lambda} \beta\right]=P^{2}$ or $\alpha^{k} \Delta_{\lambda} \alpha=\beta^{k} \Delta_{\lambda} \beta$

(ii) If $k \geq 16$, then $\left[\alpha^{k} \Delta_{\lambda} \alpha\right]\left[\beta^{k} \Delta_{\lambda} \beta\right]=P^{2}$ or $\alpha^{k} \Delta_{\lambda} \alpha=\beta^{k} \Delta_{\lambda} \beta$.

Theorem 9. Let $k \geq 16$ be an integer and $\lambda \neq 0$ be a complex number. Assume that the distinct nonconstant meromorphic mappings $\alpha$ and $\beta$ have finite order. Suppose that $\alpha$ and $\beta$ share $0, \infty C M, \alpha^{k} \Delta_{\lambda} \alpha$ and $\beta^{k} \Delta_{\lambda} \beta$ share 1 CM. If

$$
\limsup _{q \rightarrow \infty} \frac{\bar{N}\left(q, \frac{1}{\alpha(y)-1}\right)+\bar{N}\left(q, \frac{1}{\beta(y)-1}\right)}{T(q, \alpha(y))+T(q, \beta(y))}<1-\frac{3}{k^{\prime}}
$$

then one of the two cases given below holds:

(i) $f=c_{1} \beta$, where $c_{1} \neq 1$ is a constant fulfilling $c_{1}^{k+1}=1$.

(ii) For all $y \in C, \alpha(y)=\alpha(y+\lambda)$ and $\beta(y)=\beta(y+\lambda)$.

Proving Theorem 9 in Section 3, we can obtain the following interesting uniqueness results. In the complex plane, the difference polynomials of the following meromorphic mappings have the same fixed points. 
Theorem 10. Suppose that $k \geq 16$ be an integer and $\lambda \neq 0$ be a complex number. Let the distinct nonconstant meromorphic mappings $\alpha$ and $\beta$ have finite orders. Suppose that $\alpha$ and $\beta$ share $0, \infty C M, \alpha^{k}(y) \Delta_{\lambda} \alpha(y)-y$ and $\beta^{k}(y) \Delta_{\lambda} \beta(y)-y$ share 0 CM. If the inequality (4) holds, then one of the conclusions (i) and (ii) of Theorem 9 can occur.

In view of Theorem 5 and Lemma 2.9, we will derive the following results for entire mappings.

Theorem 11. Assume that $k \geq 5$ be an integer, $\lambda \neq 0$ be a complex number and $P \neq \equiv$ be a polynomial. Let the distinct transcendental meromorphic mappings $\alpha$ and $\beta$ have finite orders. Suppose that $\Delta_{\lambda} \alpha \neq \equiv$ and $\Delta_{\lambda} \beta \not \equiv 0$. Let $\alpha^{n} \Delta_{\lambda} \alpha-P$ and $\beta^{k} \Delta_{\lambda} \beta-P$ share 0 CM. Then

(i) If $k \geq 5$ and if $\alpha^{k} \Delta_{\lambda} \alpha / P$ is a Möbius transformation of $\beta^{k} \Delta_{\lambda} \beta / P$, then $\left[\alpha^{k} \Delta_{\lambda} \alpha\right]\left[\beta^{k} \Delta_{\lambda} \beta\right]=P^{2}$ or $\alpha^{k} \Delta_{\lambda} \alpha=\beta^{k} \Delta_{\lambda} \beta$.

(ii) If $n \geq 7$, then $\left[\alpha^{k} \Delta_{\lambda} \alpha\right]\left[\beta^{k} \Delta_{\lambda} \beta\right]=P^{2}$ or $\alpha^{k} \Delta_{\lambda} \alpha=\beta^{k} \Delta_{\lambda} \beta$.

The above theorem gives us the following two uniqueness theorems of entire mappings. The difference polynomials of the mentioned mappings share a nonzero constant or have the same fixed points in the plane.

Theorem 12. Suppose that $k \geq 7$ be an integer, $\lambda$ be a nonzero complex number. Let the distinct nonconstant entire mappings $\alpha$ and $\beta$ have finite order. Assume that $\alpha$ and $\beta$ share $0, \infty C M, \alpha^{k} \Delta_{\lambda} \alpha$ and $\beta^{k} \Delta_{\lambda} \beta$ share $1 C M$. Then one of the following arguments holds.

(i) $\alpha=c_{1} \beta$, where $c_{1} \neq 1$ is a constant fulfilling $c_{1}^{k+1}=1$.

(ii) For all $y \in C, \alpha(y)=\alpha(y+\lambda)$ and $\beta(y)=\beta(y+\lambda)$.

(iii) $\alpha(y)=e^{a_{1} y+a_{0}}$ and $\beta(y)=e^{-a_{1} y+b_{0}}$, where $a_{1}, a_{0}$ and $b_{0}$ are complex numbers such that $a_{1} \neq 0$ and $e^{(k+1)\left(a_{0}+b_{0}\right)}\left(e^{a_{1} \eta}-1\right)\left(e^{-a_{1} \eta}-1\right)=1$.

Theorem 13. Suppose that $k \geq 7$ be an integer and $\lambda$ be a nonzero complex number. Let the distinct nonconstant entire mappings $\alpha$ and $\beta$ have finite orders. Assume that $\alpha$ and $\beta$ share $0, \infty C M, \alpha^{k}(y) \Delta_{\lambda} \alpha(y)-y$ and $\beta^{k}(y) \Delta_{\lambda} \beta(y)-y$ share 0 CM. Then one of the conclusions (i) and (ii) of Theorem 12 holds.

\section{Preliminaries}

Building on the previous ideas of meromorphic mapping and Nevanlinna theory, this section contains the fundamental definitions, notions and results required for the further study of the subject. For more details on the concepts briefly discussed, readers are suggested to consult the papers [8-14]. Let $c \in C \cup\{\infty\}, p \in Z^{+}$and $\alpha$ be meromorphic mapping, which is not a constant. Then we give the following three definitions $[15,16]$.

Definition 2. The counting mapping of those c-points of $\alpha$ whose multiplicities are not greater than $p$ is denoted $N_{p}(q, 1 /(\alpha-c))$. The corresponding reduced counting mapping (ignoring multiplicities) is indicated by $\bar{N}_{p)}(q, 1 /(\alpha-c)) . N_{(p}(q, 1 /(\alpha-c))$ represents the counting mapping of those c-points of $\alpha$ (counted with proper multiplicities) whose multiplicities are not less than $p . B y \bar{N}_{(p}(q, 1 /(\alpha-c))$ we present the corresponding reduced counting mapping (ignoring multiplicities), where $N_{p)}(q, 1 /(\alpha-c))$, $\bar{N}_{p)}(q, 1 /(\alpha-c)), N_{(p}(q, 1 /(\alpha-c))$ and $\bar{N}_{(p}(q, 1 /(\alpha-c))$ mean $N_{p)}(q, \alpha), \bar{N}_{p)}(q, \alpha), N_{(p}(q, \alpha)$ and $\bar{N}_{(p}(q, \alpha)$ respectively, if $c=\infty$.

Definition 3. Assume that $k$ is a nonnegative integer. Let $\alpha$ be a meromorphic mapping, which is not constant. Suppose that $c$ be any value in the extended complex plane. Then we set

$$
N_{k}\left(q, \frac{1}{\alpha-c}\right)=\bar{N}\left(q, \frac{1}{\alpha-c}\right)+\bar{N}_{(2}\left(q, \frac{1}{\alpha-c}\right)+\cdots+\bar{N}_{(k}\left(q, \frac{1}{\alpha-c}\right) .
$$


Definition 4. Let $k \geq 2$ be an integer. Assume that $\alpha$ is a meromorphic mapping, which is not constant. The difference operators are defined by $\Delta_{\lambda} \alpha(y)=\alpha(y+\lambda)-\alpha(y), \Delta_{\lambda}^{k} \alpha(y)=\Delta_{\lambda}^{k-1}\left(\Delta_{\lambda} \alpha(y)\right)$, where $\lambda$ is a nonzero complex number. If $\lambda=1$, we represent $\Delta_{\lambda} \alpha(y)=\Delta \alpha(y)$. Also,

$$
\Delta_{\lambda}^{k} \alpha(y)=\sum_{j=0}^{k}\left(\begin{array}{l}
k \\
j
\end{array}\right)(-1)^{k-j} \alpha(y+j \lambda)
$$

Now we state some important lemmas. These lemmas will be used in the proof of our forthcoming results. The following first lemma is borrowed from [13] while second and third lemmas can be found in [17].

Lemma 1. In the complex plane, consider a nonconstant meromorphic mapping $\alpha$. Let $c_{0}, c_{1}, \cdots, c_{k-1}, c_{k} b e$ arbitrary constants and

$$
P(\alpha)=c_{k} \alpha(y)^{k}+c_{k-1} \alpha(y)^{k-1}+\cdots+c_{1} \alpha(y)+c_{0}
$$

where $c_{k} \neq 0$. Then

$$
m(q, P(\alpha))=k m(q, \alpha)+O(1) .
$$

Lemma 2. Let $\lambda \in C$. Consider a meromorphic mapping $\alpha$, which is not constant. If $\alpha$ is of finite order, then

$$
m\left(q, \frac{\alpha(y+\lambda)}{\alpha(y)}\right)=O\left(\frac{T(q, \alpha(y)) \log q}{q}\right)
$$

for every $q$ outside of a set $\Omega$ fulfilling

$$
\limsup _{q \rightarrow \infty} \frac{\int_{\Omega \cap[1, q)} d t / t}{\log q}=0,
$$

i.e., outside of a set $\Omega$ of zero logarithmic density. If $\rho_{2}(\alpha)=\rho_{2}<1$ and $\varepsilon>0$. Then for every $q$ outside of a finite logarithmic measure

$$
m\left(q, \frac{\alpha(y+\lambda)}{\alpha(y)}\right)=o\left(\frac{T(q, \alpha(y))}{q^{1-\rho_{2}-\varepsilon}}\right)
$$

where $\varepsilon$ is a positive number.

Lemma 3. Let $s \in R^{+}$. Consider a continuous mapping $T:[0,+\infty) \longrightarrow[0,+\infty)$, which is nondecreasing. If

$$
\limsup _{q \rightarrow \infty} \frac{\log \log T(q)}{\log q}=\zeta<1
$$

and $\delta \in(0,1-\zeta)$, i.e., the hyper-order of $T$ is strictly less than one. Then

$$
T(q+s)=T(q)+o\left(\frac{T(q)}{q^{\delta}}\right),
$$

where outside of a set of finite logarithmic measure, q runs to infinity.

For the next four lemmas see $[1,18]$. 
Lemma 4. Consider two meromorphic mappings $F$ and $G$, which are nonconstant and $G$ is a Möbius transformation of $F$. Assume that a subset $I \subset R^{+}$with its linear measure mes $I=+\infty$ exists and

$$
\bar{N}\left(q, \frac{1}{F}\right)+\bar{N}(q, F)+\bar{N}\left(q, \frac{1}{G}\right)+\bar{N}(q, G)<(\eta+o(1)) T(q, F),
$$

as $q \in I$ and $q \longrightarrow \infty$, where $\lambda<1$. If a point $y_{0} \in C$ exists in such a way that $F\left(y_{0}\right)=G\left(y_{0}\right)=1$, then $F=G$ or $F G=1$.

Lemma 5. Consider two meromorphic mappings $F$ and $G$, which are nonconstant. Let $F$ and $G$ share $1 C M$. Assume that a subset $I \subset R^{+}$with its linear measure mes $I=\infty$ exists and

$$
N_{2}(q, F)+N_{2}(q, G)+N_{2}\left(q, \frac{1}{F}\right)+N_{2}\left(q, \frac{1}{G}\right)<(\mu+o(1)) T(q),
$$

where $\mu<1, T(q)=\max \{T(q, F), T(q, G)\}$. Then $F=G$ or $F G=1$.

Lemma 6. Consider two meromorphic mappings $F$ and $G$, which are nonconstant. Let $F$ and $G$ share $1, \infty C M$. Assume that a subset $I \subset R^{+}$with its linear measure mes $I=+\infty$ exists and

$$
N_{2}\left(q, \frac{1}{F}\right)+N_{2}\left(q, \frac{1}{G}\right)+2 \bar{N}(q, F)<\lambda T(q)+S(q),
$$

as $q \in I$ and $q \longrightarrow \infty$, where $\lambda<1, T(q)=\max \{T(q, F), T(q, G)\}$ and $S(q)=o\{T(q)\}$, as $q \in I$ and $q \longrightarrow \infty$. Then $F=G$ or $F G=1$.

Lemma 7. Consider the nonconstant meromorphic mappings $\alpha_{1}, \alpha_{2}, \cdots, \alpha_{n}$. Let $\alpha_{k+1} \not \equiv 0$ be a meromorphic mapping such that $\sum_{j=1}^{k+1} f_{j}=1$. If a subset $I \subseteq R^{+}$fulfilling mes $I=\infty$ exists and

$$
\sum_{i=1}^{k+1} N\left(q, \frac{1}{\alpha_{i}}\right)+k \sum_{i=1, i \neq j}^{k+1} \bar{N}\left(q, \alpha_{i}\right)<\{\lambda+o(1)\} T\left(q, \alpha_{j}\right), \quad j=1,2, \cdots, k,
$$

as $q \longrightarrow \infty$ and $q \in I$, where $\lambda<1$, then $\alpha_{k+1}=1$.

The following lemma can be found in [19].

Lemma 8. Consider two rational mapping $\alpha$ and $\beta$, which are nonconstant. Let they share $0,1, \infty C M$. Then $\alpha=\beta$. Now let $P(y)=(a+i b) y^{k}+\cdots$ be a polynomial of degree $k \geq 1$, where $a$ and $b$ are real numbers such that $a+i b \neq 0$, and let $y=q e^{i \theta}$. Then $\operatorname{Re}\left\{(a+i b) e^{k \theta i}\right\}=a \cos k \theta-b \sin k \theta=: \delta(P, \theta)$.

The following results will be utilized to prove Theorem 9. For its proof see [20].

Lemma 9. Let $P(y)$ be a polynomial of degree $k \geq 1$, and let $\varepsilon>0$ be a given constant. Then we have

(i) If $\delta(P, \theta)>0$, then there exists an $q(\theta)>0$ such that for any $q>q(\theta)$, we have

$$
\left|e^{P\left(q e^{i \theta}\right)}\right| \geq \exp \left((1-\varepsilon) \delta(P, \theta) q^{k}\right)
$$

(ii) If $\delta(P, \theta)<0$, then there exists an $q(\theta)<0$ such that for any $q>q(\theta)$, we have

$$
\left|e^{P\left(q e^{i \theta}\right)}\right| \leq \exp \left((1-\varepsilon) \delta(P, \theta) q^{k}\right)
$$

The proof of the following lemma can be found on page 177 of [21]. 
Lemma 10. Assume that $\alpha(y)$ be an analytic mapping of $y=q^{i \theta}$, regular in the region $D$ between two straight lines making an angle $\pi / a$ at the origin and on the lines themselves. Let $|f(y)| \leq M$ on the lines, where $M>0$ be some constant, and that, as $|y| \rightarrow \infty,|\alpha(y)|=O\left(e^{q^{\beta}}\right)$, where $b<a$, uniformly in the angle. Then actually the inequality $|\alpha(y)| \leq M$ holds throughout the region $D$.

\section{Proof of Results}

In this section, we provide the proof of theorems, stated in first section.

Proof. (Theorem 4): In view of Lemmas 1 and 2 we obtain

$$
\begin{aligned}
& (k+1) T(q, \alpha(y)) \\
= & T\left(q, \alpha^{k+1}(y)\right)+O(1) \\
= & T\left(q, \frac{\alpha(y)}{\Delta_{\eta} \alpha(y)} \cdot \alpha^{k}(y) \Delta_{\lambda} \alpha(y)\right)+O(1) \\
\leq & T\left(q, \frac{\Delta_{\lambda} \alpha(y)}{\alpha(y)}\right)+T\left(q, \alpha^{k}(y) \Delta_{\lambda} \alpha(y)\right)+O(1) \\
= & m\left(q, \frac{\alpha(y+\lambda)}{\alpha(y)}-1\right)+N\left(q, \frac{\alpha(y+\lambda)}{\alpha(y)}-1\right)+T\left(q, \alpha^{k}(y) \Delta_{\lambda} \alpha(y)\right)+O(1) \\
\leq & N\left(q, \frac{1}{\alpha(y)}\right)+N(q, \alpha(y+\lambda))+T\left(q, \alpha^{k}(y) \Delta_{\eta} \alpha(y)\right)+o\left(\frac{T(q, \alpha(y))}{q^{1-\varepsilon}}\right),
\end{aligned}
$$

as $q \notin \Omega$ and $q \longrightarrow \infty$. Noting that

$$
\limsup _{q \longrightarrow \infty} \frac{\log \log N(q, \alpha(y))}{\log q} \leq \rho_{2}(\alpha)=0 .
$$

By virtue of Lemma 3 as $q \longrightarrow \infty$ and $q \notin \Omega$

$$
N(q, \alpha(y+\lambda)) \leq N(q+|\lambda|, \alpha(y))=N(q, \alpha(y))+o\left(\frac{T(q, \alpha(y))}{q^{1-\varepsilon}}\right)
$$

where $\Omega \subset(1, \infty)$ indicates a subset with logarithmic measure $\log$ mes $\Omega<\infty$. Similarly

$$
\begin{aligned}
& N(q, \beta(y+\lambda)) \leq N(q, \beta(y))+o\left(\frac{T(q, \beta(y))}{q^{1-\varepsilon}}\right), \\
& T(q, \alpha(y+\lambda)) \leq T(q, \alpha(y))+o\left(\frac{T(q, \alpha(y))}{q^{1-\varepsilon}}\right)
\end{aligned}
$$

and

$$
\bar{N}(q, \alpha(y+\lambda)) \leq \bar{N}(q, \alpha(y))+o\left(\frac{T(q, \alpha(y))}{q^{1-\varepsilon}}\right),
$$

as $q \notin \Omega$ and $q \longrightarrow \infty$. By virtue of (7) and (8) we get

$$
\begin{aligned}
(k+1) T(q, \alpha(y))+O(1) \leq & N\left(q, \frac{1}{\alpha(y)}\right)+N(q, \alpha(y))+T\left(q, \alpha^{k}(y) \Delta_{\lambda} \alpha(y)\right) \\
& +o\left(\frac{T(q, \alpha(y))}{q^{1-\varepsilon}}\right) \\
\leq & 2 T(q, \alpha(y))+T\left(q, \alpha^{k}(y) \Delta_{\lambda} \alpha(y)\right)+o\left(\frac{T(q, \alpha(y))}{q^{1-\varepsilon}}\right),
\end{aligned}
$$

i.e.,

$$
(k-1) T(q, \alpha(y)) \leq T\left(q, \alpha^{k}(y) \Delta_{\lambda} \alpha(y)\right)+o\left(\frac{T(q, \alpha(y))}{q^{1-\varepsilon}}\right)
$$


as $q \notin \Omega$ and $q \longrightarrow \infty$. On the other hand, by (10), (11) and Theorem 1.36 of [1] we get

$$
\begin{aligned}
T & \left(q, \alpha^{k}(y) \Delta_{\lambda} \alpha(y)\right) \\
\leq & \bar{N}\left(q, \alpha^{k}(y) \Delta_{\lambda} \alpha(y)\right)+\bar{N}\left(q, \frac{1}{\alpha^{k}(y) \Delta_{\lambda} \alpha(y)}\right)+\bar{N}\left(q, \frac{1}{\alpha^{k}(y) \Delta_{\lambda} \alpha(y)-P(y)}\right) \\
& +O(\log q) \\
\leq & \bar{N}(q, \alpha(y))+\bar{N}(q, \alpha(y+\lambda))+\bar{N}\left(q, \frac{1}{\alpha(y)}\right)+\bar{N}\left(q, \frac{1}{\alpha(y+\lambda)-\alpha(y)}\right) \\
& +\bar{N}\left(q, \frac{1}{\alpha^{k}(y) \Delta_{\lambda} \alpha(y)-P(y)}\right)+O(\log q) \\
\leq & 2 \bar{N}(q, \alpha(y))+\bar{N}\left(q, \frac{1}{\alpha(y)}\right)+\bar{N}\left(q, \frac{1}{\alpha(y+\lambda)-\alpha(y)}\right) \\
& +\bar{N}\left(q, \frac{1}{\alpha^{k}(y) \Delta_{\lambda} \alpha(y)-P(y)}\right)+o\left(\frac{T(q, \alpha(y))}{q^{1-\varepsilon}}\right)+O(\log q), \\
\leq & 4 T(q, \alpha(y))+T(q, \alpha(y+\lambda))+\bar{N}\left(q, \frac{1}{\alpha^{k}(y) \Delta_{\lambda} \alpha(y)-P(y)}\right) \\
& +o\left(\frac{T(q, \alpha(y))}{q^{1-\varepsilon}}\right)+O(\log q) \\
\leq & 5 T(q, \alpha(y))+\bar{N}\left(q, \frac{1}{\alpha^{k}(y) \Delta_{\lambda} \alpha(y)-P(y)}\right)+o\left(\frac{T(q, \alpha(y))}{q^{1-\varepsilon}}\right)+O(\log q),
\end{aligned}
$$

as $q \notin \Omega$ and $q \longrightarrow \infty$. From (12) and (13) we can get the conclusion of Theorem 1.1. Thus the proof stands completed.

Proof. (Theorem 8): To prove this theorem let us set

$$
F_{1}(y)=\frac{\alpha(y)^{k} \Delta_{\lambda} \alpha(y)}{P(y)}, \quad G_{1}(y)=\frac{\beta(y)^{k} \Delta_{\lambda} \alpha(y)}{P(y)} .
$$

Applying similar arguments as used in the proof of Theorem 4 one can derive (8)-(12). From (12) and the left equality of (14) yields

$$
(k-1) T(q, \alpha(y)) \leq T\left(q, F_{1}(y)\right)+o\left(\frac{T(q, \alpha(y))}{q^{1-\varepsilon}}\right)+O(\log q),
$$

as $q \notin \Omega$ and $q \longrightarrow \infty$. Similarly

$$
(k-1) T(q, g(y)) \leq T\left(q, G_{1}(y)\right)+o\left(\frac{T(q, \beta(y))}{q^{1-\varepsilon}}\right)+O(\log q),
$$

From the condition $k \geq 16$ and the condition that $\alpha, \beta$ are transcendental meromorphic functions, we can deduce from (15), (16) and Lemma 3 that $F_{1}, G_{1}$ are transcendental meromorphic mappings. Suppose that $y_{0} \in C$ is a zero of $F_{1}-1$ of multiplicity $\mu$. Then, by the condition that $P(y) \not \equiv 0$ is a polynomial we can see that $y_{0}$ is a zero of $\alpha(y)^{n} \alpha(y+\lambda)-P(y)$ of multiplicity $\mu+v$, where $v \geq 0$ is the multiplicity of $y_{0}$ as a zero of $P(y)$. Hence $y_{0}$ is a zero of $g(y)^{k} g(y+\lambda)-P(y)$ of multiplicity $\mu+v$ by the value sharing assumption. Now one sees that $y_{0}$ is a zero of $G_{1}-1$ of multiplicity $\mu$. This also works in the other direction. Therefore, $F_{1}$ and $G_{1}$ indeed share $1 \mathrm{CM}$. As the order of $\alpha$ as well as $\beta$ is finite, so (14) and Lemma 3 yields that the same is true for $F_{1}$ and $G_{1}$ as well. We now study the following two cases: 
Case 1. Consider a Möbius transformation $F_{1}$ of $G_{1}$. By virtue of the Valiron-Mokhon'ko lemma [22] and (3.8) we obtain

$$
\begin{aligned}
T\left(q, F_{1}(y)\right) & =T\left(q, \alpha(y)^{k} \Delta_{\lambda} \alpha(y)\right)+O(\log q) \\
& =T\left(q, \beta(y)^{k} \Delta_{\lambda} \beta(y)\right)+O(\log q) \\
& =T\left(q, G_{1}(y)\right)+O(1) .
\end{aligned}
$$

From Theorem 4 we get

$$
(k-6) T(q, \alpha) \leq \bar{N}\left(q, \frac{1}{\alpha^{k} \Delta_{\lambda} \alpha-P}\right)+o\left(\frac{T(q, \alpha)}{q^{1-\varepsilon}}\right)+O(1) .
$$

The inequality (18) together with Lemma 3 and the condition that $\alpha(y)^{k} \Delta_{\lambda} \alpha(y)-P(y)$ and $\beta(y)^{k} \Delta_{\lambda} \beta(y)-P(y)$ share $0 \mathrm{CM}$ gives

$$
\begin{aligned}
& (k-6) T(q, \alpha(y)) \\
\leq & \bar{N}\left(q, \frac{1}{\beta^{k}(y) \Delta_{\lambda} \beta(y)-P(y)}\right)+o\left(\frac{T(q, \alpha(y))}{q^{1-\varepsilon}}\right)+O(1) \\
\leq & T\left(q, G_{1}(y)\right)+o\left(\frac{T(q, \alpha(y))}{q^{1-\varepsilon}}\right)+O(\log q)
\end{aligned}
$$

as $q \longrightarrow \infty$ and $q \notin \Omega$. In a similar way

$$
\begin{aligned}
& (k-6) T(q, \beta(y)) \\
\leq & \bar{N}\left(q, \frac{1}{\alpha^{k}(y) \Delta_{\lambda} \alpha(y)-P(y)}\right)+o\left(\frac{T(q, \beta(y))}{q^{1-\varepsilon}}\right)+O(1) \\
\leq & T\left(q, F_{1}(y)\right)+o\left(\frac{T(q, \beta(y))}{q^{1-\varepsilon}}\right)+O(\log q),
\end{aligned}
$$

as $q \notin \Omega$ and $q \longrightarrow \infty$. From Lemma 2 and the left equality of (14) we have

$$
\begin{aligned}
m\left(q, G_{1}\right) & \leq m\left(q, \beta(y)^{k} \Delta_{\lambda} \beta(y)\right)+m\left(q, \frac{1}{P(y)}\right) \\
& \leq m\left(q, \beta(y)^{k+1}\left(\frac{\beta(y+\lambda)}{\beta(y)}-1\right)\right)+O(1) \\
& \leq m\left(q, \beta(y)^{k+1}\right)+m\left(q, \frac{\beta(y+\lambda)}{\beta(y)}-1\right)+O(1) \\
& \leq(k+1) m(q, \beta(y))+m\left(q, \frac{\beta(y+\lambda)}{\beta(y)}\right)+O(1) \\
& \leq(k+1) m(q, \beta(y))+o\left(\frac{T(q, \beta(y))}{q^{1-\varepsilon}}\right)
\end{aligned}
$$

as $q \notin \Omega$ and $q \longrightarrow \infty$. By similar arguments as used in the proof of Theorem 4 we derive (9). From (9) and the left equality of (14) we obtain

$$
\begin{aligned}
N\left(q, G_{1}\right) & \leq N\left(q, \beta(y)^{k} \Delta_{\lambda} \beta(y)\right)+N\left(q, \frac{1}{P(y)}\right) \\
& \leq k N(q, \beta(y))+N(q, \beta(y+\lambda)-\beta(y))+O(\log q) \\
& \leq(k+2) N(q, \beta(y))+o\left(\frac{T(q, \beta(y))}{q^{1-\varepsilon}}\right)+O(\log q),
\end{aligned}
$$


as $q \longrightarrow \infty$ and $q \notin \Omega$. Equations (21) and (22) yield

$$
\begin{aligned}
T\left(q, G_{1}(y)\right) & \leq k T(q, \beta(y))+N(q, \beta(y))+o\left(\frac{T(q, \beta(y))}{q^{1-\varepsilon}}\right)+O(\log q) \\
& \leq(k+1) T(q, \beta(y))+o\left(\frac{T(q, \beta(y))}{q^{1-\varepsilon}}\right)+O(\log r),
\end{aligned}
$$

as $q \longrightarrow \infty$ and $q \notin \Omega$. Similarly

$$
T\left(q, F_{1}(y)\right) \leq(k+1) T(q, \alpha(y))+o\left(\frac{T(q, \alpha(y))}{q^{1-\varepsilon}}\right)+O(\log q),
$$

as $q \notin \Omega$ and $q \longrightarrow \infty$. From (23), (24), the condition $k \geq 16$, Definition 2 and Lemma 1.1.2 of [23] we obtain

$$
\rho(\alpha) \leq \rho\left(G_{1}\right) \leq \rho(\beta)
$$

Similarly, from (20) and (25) we have

$$
\rho(\beta) \leq \rho\left(F_{1}\right) \leq \rho(\alpha)
$$

From (25) and (26) we get

$$
\rho(\alpha)=\rho(\beta)=\rho\left(F_{1}\right)=\rho\left(G_{1}\right) .
$$

From (10) and (14) we derive

$$
\begin{aligned}
& \bar{N}\left(q, F_{1}(y)\right)+\bar{N}\left(q, \frac{1}{F_{1}(y)}\right) \\
\leq & \bar{N}(q, \alpha(y))+\bar{N}(q, \alpha(y+\lambda))+\bar{N}\left(q, \frac{1}{\alpha(y)}\right)+\bar{N}\left(q, \frac{1}{\alpha(y+\lambda)-\alpha(y)}\right) \\
& +O(\log q) \\
\leq & 3 T(q, \alpha(y))+2 T(q, \alpha(y+\lambda))+O(\log q) \\
\leq & 5 T(q, \alpha(y))+o\left(\frac{T(q, \alpha(y))}{q^{1-\varepsilon}}\right)+O(\log q),
\end{aligned}
$$

as $q \notin \Omega$ and $q \longrightarrow \infty$. Similarly

$$
\bar{N}\left(q, G_{1}(y)\right)+\bar{N}\left(q, \frac{1}{G_{1}(y)}\right) \leq 5 T(q, \beta(y))+o\left(\frac{T(q, \beta(y))}{q^{1-\varepsilon}}\right)+O(\log q),
$$

as $q \notin \Omega$ and $q \longrightarrow \infty$. Applying similar arguments as utilized in the proof of Theorem 1.1 we can derive (13). From (15) and (16) we get

$$
T(q, \alpha(y)) \leq \frac{1}{k-1} T\left(q, F_{1}(y)\right)+o\left(\frac{T(q, \alpha(y))}{q^{1-\varepsilon}}\right)
$$

and

$$
T(q, \beta(y)) \leq \frac{1}{k-1} T\left(q, G_{1}(y)\right)+o\left(\frac{T(q, \beta(y))}{q^{1-\varepsilon}}\right),
$$

as $q \notin \Omega$ and $q \longrightarrow \infty$. From (21), (27)-(31) we derive

$$
\begin{aligned}
& \bar{N}\left(q, \frac{1}{F_{1}}\right)+\bar{N}\left(q, F_{1}\right)+\bar{N}\left(q, \frac{1}{G_{1}}\right)+\bar{N}\left(q, G_{1}\right) \\
\leq & \frac{5}{k-1}\left\{T\left(q, F_{1}\right)+T\left(q, G_{1}\right)\right\}+o\left(\frac{T(q, \alpha(y))}{q^{1-\varepsilon}}\right)+o\left(\frac{T(q, \beta(y))}{q^{1-\varepsilon}}\right)+O(\log q) \\
= & \frac{10}{k-1} T\left(q, F_{1}\right)(1+o(1)),
\end{aligned}
$$


as $q \notin \Omega$ and $q \longrightarrow \infty$. This together with (32), Lemma 4 and the condition $k \geq 12$ gives $F_{1} G_{1}=1$ or $F_{1}=G_{1}$. This proves the conclusion (i) of Theorem 8

Case 2. Suppose that $k \geq 16$. In the same manner as in the proof of Case 1 we can get (30) and (31). From (10) and (14) we have

$$
\begin{aligned}
& N_{2}\left(r, F_{1}(y)\right)+N_{2}\left(q, \frac{1}{F_{1}(y)}\right) \\
\leq & 2 \bar{N}(q, \alpha(y))+N(q, \alpha(y+\lambda))+2 \bar{N}\left(q, \frac{1}{\alpha(y)}\right)+N\left(q, \frac{1}{\alpha(y+\lambda)-\alpha(y)}\right) \\
& +O(\log q) \\
\leq & 5 T(q, \alpha(y))+2 T(q, \alpha(y+\lambda))+O(\log q) \\
\leq & 7 T(q, \alpha(y))+o\left(\frac{T(q, \alpha(y))}{q^{1-\varepsilon}}\right)+O(\log q)
\end{aligned}
$$

and

$$
N_{2}\left(r, G_{1}(y)\right)+N_{2}\left(q, \frac{1}{G_{1}(y)}\right) \leq 7 T(q, \beta(y))+o\left(\frac{T(q, \beta(y))}{q^{1-\varepsilon}}\right)+O(\log q),
$$

as $q \notin \Omega$ and $q \longrightarrow \infty$. From (30), (31), (33) and (34) we have

$$
N_{2}\left(q, F_{1}(y)\right)+N_{2}\left(q, \frac{1}{F_{1}(y)}\right) \leq \frac{7}{k-1} T\left(q, F_{1}(y)\right)+o\left(\frac{T(q, \alpha(y))}{q^{1-\varepsilon}}\right)+O(\log r)
$$

and

$$
N_{2}\left(q, G_{1}(y)\right)+N_{2}\left(q, \frac{1}{G_{1}(y)}\right) \leq \frac{7}{k-1} T\left(q, G_{1}(y)\right)+o\left(\frac{T(q, \beta(y))}{q^{1-\varepsilon}}\right)+O(\log q),
$$

as $q \notin \Omega$ and $q \longrightarrow \infty$. From (27), (35) and (36) we have

$$
N_{2}\left(q, F_{1}(y)\right)+N_{2}\left(q, \frac{1}{F_{1}(y)}\right)+N_{2}\left(q, G_{1}(y)\right)+N_{2}\left(q, \frac{1}{G_{1}(y)}\right) \leq \frac{14}{k-1} T_{1}(q)(1+o(1)),
$$

as $q \notin \Omega$ and $q \longrightarrow \infty$, where $T_{1}(q)=\max \left\{T\left(q, F_{1}\right), T\left(q, G_{1}\right)\right\}$. From (37), Lemma 5 and the condition $k \geq 16$ we have $F_{1}=G_{1}$ or $F_{1} G_{1}=1$. This reveals the conclusion (ii) of Theorem 8 . Thus the proof stands completed.

Proof. (Theorem 9): This theorem is proved by considering the below two cases:

Case 1. Let one of $\alpha$ and $\beta$, say $\alpha$, is a rational mapping. Then, $\beta$ is a rational function. In fact, if $\beta$ is a transcendental meromorphic mapping, then, in the same manner as in the proof of (20) we can get from the assumption of Theorem 9 that

$$
\begin{aligned}
(k-6) T(q, \beta(y)) & \leq \bar{N}\left(q, \frac{1}{\alpha^{k}(y) \Delta_{\lambda} \alpha(y)-1}\right)+o\left(\frac{T(q, \beta(y))}{q^{1-\varepsilon}}\right)+O(1) \\
& \leq o\left(\frac{T(q, \beta(y))}{q^{1-\varepsilon}}\right)+O(\log q),
\end{aligned}
$$

as $q \longrightarrow \infty$ and $q \notin \Omega$. From $k \geq 16$ and (38) we can deduce $T(q, \beta(y))=O(\log q)$, as $q \notin \Omega$ and $q \longrightarrow \infty$. This implies that $\beta$ is a rational function, which is impossible. Therefore, by virtue of the condition that $\alpha$ and $\beta$ share $0, \infty \mathrm{CM}$ we derive

$$
\alpha=c_{1} \beta
$$


where $c$ is some nonzero complex number. Thus

$$
\alpha^{k} \Delta_{\lambda} \alpha=c_{1}^{k+1} \beta^{k} \Delta_{\lambda} \beta
$$

Suppose that $\Delta_{\lambda} \alpha=0$. Then, if $\alpha(y)$ has a zero at some point $y_{0}$, then $\alpha(y)$ has a zero at $y_{0}+\lambda$ by $\Delta_{\lambda} \alpha=0$. Continuing, $\alpha\left(y_{0}+2 \lambda\right)=0, \alpha\left(y_{0}+3 \lambda\right)=\infty$, and so on. Therefore, $\alpha(y)$ would have infinitely many zeros, which is impossible. Similarly, one can obtain a contradiction, if $\alpha(y)$ has a pole at some point $y_{1} \in C$. Therefore, $\Delta_{\lambda} \alpha \neq \equiv 0$, and so $\Delta_{\lambda} \beta \not \equiv 0$ by (40). Combining this with (40) and the assumption that $\alpha$ and $\beta$ share $0, \infty \mathrm{CM}$, we find that $\alpha^{k} \Delta_{\lambda} \alpha$ and $\beta^{k} \Delta_{\lambda} \beta$ share $0, \infty \mathrm{CM}$. This together with Lemma 8 and the assumption that $\alpha^{k} \Delta_{\lambda} \alpha$ and $\beta^{k} \Delta_{\lambda} \beta$ share $1 \mathrm{CM}$ gives

$$
\alpha^{k} \Delta_{\lambda} \alpha=\beta^{k} \Delta_{\lambda} \beta
$$

From (40) and (41) we have $c_{1}^{k+1}=1$. Together with (39), this proves the conclusion (i) of Theorem 9.

Case 2. Consider two transcendental meromorphic mappings $\alpha$ and $\beta$. Then, from Theorem 8 we have $\left[\alpha^{k} \Delta_{\lambda} \alpha\right]\left[\beta^{k} \Delta_{\lambda} \beta\right]=1$ or $\alpha^{k} \Delta_{\lambda} \alpha=\beta^{k} \Delta_{\lambda} \beta$. The present case is divided in the below two subcases:

Subcase 2.1. Let

$$
\left[\alpha^{k} \Delta_{\lambda} \alpha\right]\left[\beta^{k} \Delta_{\lambda} \beta\right]=1
$$

One can derive (8) and (9) in a similar manner as in the proof of Theorem 4. Combining this with Definition 4, Lemma 2 and the assumption that $\alpha$ and $\beta$ share $0, \infty \mathrm{CM}$ we deduce

$$
\begin{aligned}
2 n \bar{N}\left(q, \frac{1}{\alpha(y)}\right) & =k \bar{N}\left(q, \frac{1}{\alpha(y)}\right)+k \bar{N}\left(q, \frac{1}{\beta(y)}\right) \\
& \leq N(q, \alpha(y+\lambda))+N(q, \beta(y+\lambda)) \\
& =2 N(q, \alpha(y+\lambda)) \\
& \leq 2 N(q, \alpha(y))+o\left(\frac{T(q, \alpha(y))}{q^{1-\varepsilon}}\right) \\
& \leq 2 T(q, \alpha(y))+o\left(\frac{T(q, \alpha(y))}{q^{1-\varepsilon}}\right)
\end{aligned}
$$

and

$$
\begin{aligned}
2 k \bar{N}(q, \alpha(y))= & k \bar{N}(q, \alpha(y))+k \bar{N}(q, \beta(y)) \\
\leq & N\left(q, \frac{1}{\alpha(y+\lambda)-\alpha(y)}\right)+N\left(q, \frac{1}{\beta(y+\lambda)-\beta(y)}\right) \\
\leq & T(q, \alpha(y+\lambda)-\alpha(y))+T(q, \beta(y+\lambda)-\beta(y)) \\
\leq & m\left(q, \alpha(y)\left(\frac{\alpha(y+\lambda)}{\alpha(y)}-1\right)\right)+N(q, \alpha(y+\lambda))+N(q, \alpha(y)) \\
& +m\left(q, \beta(y)\left(\frac{\beta(y+\lambda)}{\beta(y)}-1\right)\right)+N(q, \beta(y+\lambda))+N(q, \beta(y)) \\
\leq & m(q, \alpha(y))+2 N(q, \alpha(y))+m(q, \beta(y))+2 N(q, \beta(y)) \\
& +o\left(\frac{T(q, \alpha(y))}{q^{1-\varepsilon}}\right)+o\left(\frac{T(q, \beta(y))}{q^{1-\varepsilon}}\right) \\
\leq & 2 T(q, \alpha(y))+2 T(q, \beta(y))+o\left(\frac{T(q, \alpha(y))}{q^{1-\varepsilon}}\right)+o\left(\frac{T(q, \beta(y))}{q^{1-\varepsilon}}\right)
\end{aligned}
$$

i.e.,

$$
\begin{gathered}
\bar{N}\left(q, \frac{1}{\alpha(y)}\right) \leq \frac{1}{k} T(q, \alpha(y))+o\left(\frac{T(q, \alpha(y))}{q^{1-\varepsilon}}\right) \\
\bar{N}(q, \alpha(y)) \leq \frac{1}{k} T(q, \alpha(y))+\frac{1}{k} T(q, \beta(y))+o\left(\frac{T(q, \alpha(y))}{q^{1-\varepsilon}}\right)+o\left(\frac{T(q, \beta(y))}{q^{1-\varepsilon}}\right),
\end{gathered}
$$


as $q \notin \Omega$ and $q \longrightarrow \infty$. By (43), (44) and the second fundamental theorem we obtain

$$
\begin{aligned}
T(q, \alpha(y)) \leq & \bar{N}(q, \alpha(y))+\bar{N}(q, \alpha(y))+\bar{N}\left(q, \frac{1}{\alpha(y)-1}\right)+O(\log q) \\
\leq & \frac{2}{k} T(q, \alpha(y))+\frac{1}{k} T(q, \beta(y))+\bar{N}\left(q, \frac{1}{\alpha(y)-1}\right)+O(\log q) \\
& +o\left(\frac{T(q, \alpha(y))}{q^{1-\varepsilon}}\right)+o\left(\frac{T(q, \beta(y))}{q^{1-\varepsilon}}\right)+O(\log q),
\end{aligned}
$$

as $q \notin \Omega$ and $q \longrightarrow \infty$. Similarly

$$
\begin{aligned}
T(q, \beta(y)) \leq & \frac{2}{k} T(q, \beta(y))+\frac{1}{k} T(q, \alpha(y))+\bar{N}\left(q, \frac{1}{\beta(y)-1}\right) \\
& +o\left(\frac{T(q, \alpha(y))}{q^{1-\varepsilon}}\right)+o\left(\frac{T(q, \beta(y))}{q^{1-\varepsilon}}\right)+O(\log q),
\end{aligned}
$$

as $q \notin \Omega$ and $q \longrightarrow \infty$. From (45) and (46) we have

$$
\begin{aligned}
T(q, \alpha(y))+T(q, \beta(y)) \leq & \frac{3}{k} T(q, \beta(y))+\frac{3}{k} T(q, \alpha(y))+\bar{N}\left(q, \frac{1}{\alpha(y)-1}\right) \\
& +\bar{N}\left(q, \frac{1}{\beta(y)-1}\right)+o\left(\frac{T(q, \alpha(y))}{q^{1-\varepsilon}}\right)+o\left(\frac{T(q, \beta(y))}{q^{1-\varepsilon}}\right) \\
& +O(\log q),
\end{aligned}
$$

as $q \notin \Omega$ and $q \longrightarrow \infty$. Applying similar arguments as utilized in the proof of (27) we determine $\rho(f)=\rho(\beta)<\infty$. This together with (47) gives

$$
\limsup _{r \rightarrow \infty} \frac{\bar{N}\left(q, \frac{1}{\alpha(y)-1}\right)+\bar{N}\left(q, \frac{1}{\beta(y)-1}\right)}{T(q, \alpha(y))+T(q, \beta(y))} \geq 1-\frac{3}{k^{\prime}}
$$

which contradicts the assumption (4).

Subcase 2.2. Suppose that

$$
\alpha^{k} \Delta_{\lambda} \alpha=\beta^{k} \Delta_{\lambda} \beta
$$

In view of the hypothesis that $\alpha$ and $\beta$ share $0, \infty \mathrm{CM}$ we get

$$
\frac{\alpha}{\beta}=e^{P_{1}}
$$

where $\alpha$ is an entire function. Noting that $\rho(\alpha)=\rho(\beta):=\rho<\infty$, we can get from (50) that $\rho\left(e^{P_{1}}\right) \leq \rho$, and so $P_{1}$ is a polynomial with degree $\leq \rho$. Suppose that $P_{1}$ is some constant, then $e^{P_{1}}$ is some nonzero constant, say $e^{P_{1}}=c_{2}$. Thus from (49) and (50) we get

$$
\left(c_{2}^{k+1}-1\right) \beta^{k} \Delta_{\lambda} \beta=0 .
$$

If $\Delta_{\lambda} \beta=0$, then we can get the conclusion (ii) from (49). Next we suppose that $\Delta_{\lambda} \beta \not \equiv 0$, and so we have from (51) that $c_{2}^{k+1}=1$, which together with (50) and $e^{P_{1}}=c_{2}$ reveals the conclusion (ii) of Theorem 9. Suppose that $P_{1}$ is a nonzero polynomial. Then $P_{1}(y)=\left(a_{1}+i b_{1}\right) y^{k}+\cdots$ be a polynomial of degree $k_{1} \geq 1$, where $a_{1}$ and $b_{1}$ are real numbers such that $a_{1}+i b_{1} \neq 0$. By (49) and (50) we have

$$
\frac{\beta(y+\lambda)}{\beta(y)}=\frac{e^{(k+1) P_{1}(y)}-1}{e^{k P_{1}(y)+P_{1}(y+\eta)}-1} .
$$


Given a positive number $\varepsilon$, we set

$$
T_{\varepsilon}=\bigcup_{j=0}^{k_{1}-1}\left\{y:\left|\arg y-\theta_{j}\right|<\varepsilon\right\},
$$

where

$$
\theta_{j}=\left(\frac{2 j}{k_{1}}+\frac{1}{2 k_{1}}\right) \pi-\frac{\theta_{k_{1}}}{k_{1}}, \quad 0 \leq j \leq k_{1}-1,
$$

such that

$$
\alpha \cos k_{1} \theta_{j}-\beta \sin k_{1} \theta_{j}=0, \quad 0 \leq j \leq k_{1}-1 .
$$

By (52)-(55) and Lemma 9 we can find that

$$
\lim _{|y| \rightarrow \infty,|y| \notin T_{\varepsilon}} \frac{\beta(y+\lambda)}{\beta(y)}=0 .
$$

Hence from (55), Lemma 10 and Liouville's Theorem we can find that $\beta(y+\lambda) / \beta(y)$ is a constant. Therefore

$$
\frac{\beta(y+\lambda)}{\beta(y)}=c_{3},
$$

where $c_{3}$ is a nonzero constant. Similarly

$$
\frac{\alpha(y+\lambda)}{\alpha(y)}=c_{4}
$$

where $c_{4}$ is a nonzero constant. If one of $c_{3}$ and $c_{4}$ is equal to 1 , then we can get the conclusion (ii) from (57), (58) and (49). Next we suppose that $c_{3} \neq 1$ and $c_{4} \neq 1$. By substituting (57) and (58) into (49) we have $\alpha^{k+1}=\frac{c_{3}-1}{c_{4}-1} \cdot \beta^{k+1}$, and so

$$
\alpha=c_{5} \beta,
$$

where $c_{5}$ is a constant satisfying $c_{5}^{k+1}=\frac{c_{3}-1}{c_{4}-1}$. Again from (59) and (49) we deduce $c_{5} \neq 1$ and $c_{5}^{k+1}=1$, which reveals the conclusion (i) of Theorem 9. The proof stands completed.

Proof. (Theorem 10): To prove the current theorem, we set

$$
F_{2}(y)=\frac{\alpha(y)^{k} \Delta_{\lambda} \alpha(y)}{y}, \quad G_{2}(y)=\frac{\beta(y)^{n} \Delta_{\lambda} \beta(y)}{y} .
$$

Then, applying similar arguments as utilized in the proof of Theorem 9 we can find that $F_{2}$ and $G_{2}$ share $1 \mathrm{CM}$. We consider the following two cases:

Case 1. Let one of $\alpha$ and $\beta$, say $\alpha$ is a nonconstant rational mapping. Then $\beta$ is also a nonconstant rational mapping. In fact, if $\beta$ is a transcendental meromorphic mapping, then we can derive in the same manner as in the proof of (20) that

$$
\begin{aligned}
& (k-6) T(q, \beta(y)) \\
\leq & \bar{N}\left(q, \frac{1}{\alpha^{k}(y) \Delta_{\lambda} \alpha(y)-y}\right)+o\left(\frac{T(q, \beta(y))}{q^{1-\varepsilon}}\right)+O(1) \\
\leq & T\left(q, F_{2}(y)\right)+o\left(\frac{T(q, \beta(y))}{q^{1-\varepsilon}}\right)+\log q+O(1) \\
= & o\left(\frac{T(q, \beta(y))}{q^{1-\varepsilon}}\right)+O(\log q),
\end{aligned}
$$


as $q \notin \Omega$ and $q \longrightarrow \infty$. From (61) and the the assumption $k \geq 16$ we can deduce that $\beta$ is a nonconstant rational mapping, this is impossible. Therefore, from (60) we can see that $F_{2}$ and $G_{2}$ are rational mapping. Next we prove that $F_{2}$ and $G_{2}$ are nonconstant rational mappings. In fact, if one of $F_{2}$ and $G_{2}$ is a constant, say $F_{2}=c_{6}$, where $c_{6}$ is a finite complex number, then we can get from the first equality of (60) that

$$
\alpha(y)^{k} \Delta_{\lambda} \alpha(y)=c_{6} y
$$

Set

$$
\alpha(y)=\frac{P_{2}}{P_{3}}
$$

where $P_{2}$ and $P_{3}$ are nonzero relatively prime polynomials. Noting that at least one of $P_{2}$ and $P_{3}$ is not a constant. Applying similar arguments as used in the proof of Theorem 9, we determine $\Delta_{\eta} \alpha(y) \not \equiv 0$. Therefore, we can get from (62), (63) and the standard Valiron-Mokhonko lemma [22] that

$$
\begin{aligned}
& \left.k \max \left\{\operatorname{deg}\left(P_{2}(y)\right), \operatorname{deg}\left(P_{3}\right)(y)\right)\right\} \log q+O(1) \\
= & k T(q, \alpha(y)) \\
\leq & T\left(q, \frac{P_{2}(y)}{P_{3}(y)}-\frac{P_{2}(y+\lambda)}{P_{3}(y+\eta)}\right)+\log r+O(1) \\
\leq & T\left(q, \frac{P_{2}(y)}{P_{3}(y)}\right)+T\left(q, \frac{P_{2}(y+\eta)}{P_{3}(y+\lambda)}\right)+O(1) \\
= & \left.2 \max \left\{\operatorname{deg}\left(P_{2}(y)\right), \operatorname{deg}\left(P_{3}\right)(y)\right)\right\} \log q+O(1),
\end{aligned}
$$

which implies that $k \leq 2$, this contradicts the assumption $k \geq 16$. Therefore, $F_{2}$ and $G_{2}$ are nonconstant rational functions. Combining this with (60) and the assumption that $\alpha$ and $\beta$ share $0, \infty \mathrm{CM}$ and the assumption that $F_{2}$ and $G_{2}$ share $1 \mathrm{CM}$, we have (39) and (40). Thus the conclusion (i) of Theorem 9 is proved.

Case 2. Let $\alpha$ and $\beta$ be transcendental meromorphic mappings. Then, by virtue of Theorems 8 and 10 we determine $\left[\alpha^{k} \Delta_{\lambda} \alpha\right]\left[\beta^{k} \Delta_{\lambda} \beta\right]=1$ or $\alpha^{k} \Delta_{\lambda} \alpha=\beta^{k} \Delta_{\lambda} \beta$. If $\alpha^{k} \Delta_{\lambda} \alpha=\beta^{k} \Delta_{\lambda} \beta$. Then by using similar arguments as utilized in Subcase 2.2 of the proof of Theorem 9 we can get the conclusions (i) and (ii). Next we suppose that $\left[\alpha^{k} \Delta_{\lambda} \alpha\right]\left[\beta^{k} \Delta_{\lambda} \beta\right]=1$. Noting that $T(q, y)=o\{T(q, \alpha)\}$ and $T(q, y)=o\{T(q, \beta)\}$, we can get in the same manner as in the proofs of (43) and (44) of Theorem 9 that

$$
\bar{N}\left(q, \frac{1}{\alpha(y)}\right) \leq \frac{1}{k} T(q, \alpha(y))+o\left(\frac{T(q, \alpha(y))}{q^{1-\varepsilon}}\right)+O(\log q)
$$

and

$$
\bar{N}(q, \alpha(y)) \leq \frac{1}{k} T(q, \alpha(y))+\frac{1}{k} T(q, \beta(y))+o\left(\frac{T(q, \alpha(y))}{q^{1-\varepsilon}}\right)+o\left(\frac{T(q, \beta(y))}{q^{1-\varepsilon}}\right)+O(\log q)
$$

as $q \notin \Omega$ and $q \longrightarrow \infty$, and so we can get (45) and (46). From (45) and (46) we have (47), and so we have (48), which contradicts (4). Thus the proof stands completed.

Proof. (Theorem 12): Let one of $\alpha$ and $\beta$ are nonconstant polynomial. Then, by using similar arguments as utilized in Case 1 of the proof of Theorem 9 we have the conclusion (i) of Theorem 9. Now assume that $\alpha$ and $\beta$ are transcendental entire functions. Then, by Theorem 11 and the assumptions of Theorem 12 we have $\left[\alpha^{k} \Delta_{\lambda} \alpha\right]\left[\beta^{k} \Delta_{\lambda} \beta\right]=1$ or $\alpha^{k} \Delta_{\lambda} \alpha=\beta^{k} \Delta_{\lambda} \beta$. Suppose that $\alpha^{k} \Delta_{\lambda} \alpha=\beta^{k} \Delta_{\lambda} \beta$. Then, by using similar arguments as utilized in Case 1 and Subcase 2.2 of the proof of Theorem 9 we can get the conclusions (i) and (ii) of Theorem 12. Suppose that

$$
\left[\alpha^{k} \Delta_{\lambda} \alpha\right]\left[\beta^{k} \Delta_{\lambda} \beta\right]=1
$$


Combining (66) with the assumption that $\alpha$ and $\beta$ are entire functions sharing $0 \mathrm{CM}$, we have

$$
\alpha=e^{f}, \quad \alpha=e^{g},
$$

where $f$ and $g$ are nonconstant polynomials. By substituting (67) into (66) we have

$$
e^{k f(y)+k g(y)}\left[e^{f(y+\lambda)}-e^{f(y)}\right]\left[e^{g(y+\lambda)}-e^{g(y)}\right]=1
$$

for all $y \in C$. By (68) we have

$$
e^{f(y+\lambda)}-e^{f(y)}=e^{\gamma(y)}
$$

for all $y \in C$, where $\gamma$ is a polynomial. By (69) and Lemma 7 we can find that $e^{f(y+\eta)-f(y)} \neq 1$ is a constant. Similarly $e^{g(y+\lambda)-g(y)} \neq 1$ is also a constant. Set

$$
f(y)=c_{m} y^{m}+c_{m-1} y^{m-1}+\cdots+c_{1} y+a_{0}
$$

where $c_{m} \neq 0, c_{m-1}, \cdots, c_{1}, c_{0}$ are complex numbers. Suppose that $m \geq 2$. Then

$$
f(y+\lambda)-f(y)=m b_{m} y^{m-1}+b_{m-2} y^{m-2}+\cdots b_{1} y+b_{0}
$$

where $b_{m-2}, b_{m-3}, \cdots, b_{1}, b_{0}$ are complex numbers. Noting that $c_{m} \neq 0$, we can find from (71) that $f(y+\lambda)-f(y)$ is not a constant, which contradicts the fact that $e^{f(y+\lambda)-f c(y)} \neq 1$ is a constant. Therefore $m=1$. Combining this with (68) and (70), we can deduce the conclusion (iii) of Theorem 12. This proves Theorem 12.

Proof. (Theorem 13): We set the equalities given in (60). Suppose that one of $\alpha$ and $\beta$ are nonconstant polynomial. Then, by using similar arguments as utilized in Case 1 of the proof of Theorem 10 we have the conclusion (i) of Theorem 12 from (60). Next assume that $\alpha$ and $\beta$ are transcendental entire mappings. Then, by (60), Theorem 11 and the assumptions of Theorem 13 we have $\left[\alpha^{n} \Delta_{\lambda} \alpha\right]\left[\beta^{n} \Delta_{\lambda} \beta\right]=z^{2}$ or $\alpha^{k} \Delta_{\lambda} \alpha=\beta^{k} \Delta_{\lambda} \beta$. Suppose that $\alpha^{k} \Delta_{\lambda} \alpha=\beta^{k} \Delta_{\lambda} \beta$. Then, in the same manner as in Case 1 and Subcase 2.2 of Theorem 9 we can get the conclusions (i) and (ii) of Theorem 12 . Suppose that

$$
\left[\alpha^{k} \Delta_{\lambda} \alpha\right]\left[\beta^{k} \Delta_{\lambda} \beta\right]=y^{2}
$$

Combining (71) with $k \geq 7$ and the assumption that $\alpha$ and $\beta$ are entire mappings sharing $0 \mathrm{CM}$, we have (67). By substituting (67) into (72) we have

$$
e^{k f(y)+k g(y)}\left[e^{f(y+\lambda)}-e^{f(y)}\right]\left[e^{g(y+\lambda)}-e^{g(y)}\right]=y^{2}
$$

for all $y \in C$. From (73) we can find that at least one of $e^{f(y+\lambda)-f(y)}-1$ and $e^{g(y+\lambda)-g(y)}-1$, say $e^{f(y+\lambda)-f(y)}-1$ has a zero of $y=0$, and so $e^{f(y+\lambda)-f(y)}$ is transcendental entire mapping, which implies that $e^{f(y+\lambda)-f(y)}-1$ has infinitely many zeros in the complex plane. But, from (73) we can find that $e^{f(y+\lambda)-f(y)}-1$ at most has one zero of $y=0$, this is a contradiction. Thus the proof stands completed.

\section{Conclusions}

In the present article, we have proved several important results for value distribution of meromorphic mappings. It has been shown that the difference polynomials of the mentioned mappings have the same fixed points or share nonzero values. We have provided examples that the previous work of Laine and Yang need generalization. The results have been derived in more general domains. Several uniqueness results of meromorphic mapping have been explored. The research work of Qi, Yang and Liu has been generalized. The current work opens several new research directions. For instance, from Corollary 1, Corollary 2 and Example 3 we give the following problem: 
Problem 1. What can be said about the conclusion of Corollary 1 , if $2 \leq n \leq 6$ ?

From Theorems 12 and 13 we pose the following problem.

Problem 2. What can be said about the conclusions of Theorems 12 and 13 , if $2 \leq n \leq 6$ ?

We hope the techniques used in the present paper will play a key role to provide a framework for the concepts briefly discussed.

Acknowledgments: The financial support of TWAS-UNESCO Associateship-Ref. 3240290714 at Centro de Investigación en Matemáticas, A.C. (CIMAT) Jalisco S/N Valenciana A.P. 40236000 Guanajuato, GTO Mexico, is deeply appreciated and acknowledged. We are very grateful to NUST research directorate for providing publication charges and awards.

Author Contributions: All of the authors provided equal contributions to the paper.

Conflicts of Interest: The authors declare that there is no conflict of interest regarding the publication of this paper.

\section{References}

1. Yang, C.C.; Yi, H.X. Uniqueness Theory of Meromorphic Functions; Kluwer Academic Publishers: Dordrecht, The Netherlands, 2003.

2. Clunie, J. On a result of Hayman. J. Lond. Math. Soc. 1967, 42, 389-392.

3. Hayman, W.K. Picard values of meromorphic functions and their derivatives. Ann. Math. 1959, 70, 9-42.

4. Laine, I.; Yang, C.C. Value distribution of difference polynomials. Proc. Jap. Acad. Ser. A 2007, 83, 148-151.

5. Liu, K.; Yang, L.Z. Value distribution of the difference operator. Arch. Math. 2009, 92, 270-278.

6. Xu, J.F.; Yi, H.X. Uniqueness of entire functions and differential polynomials. Bull. Korean Math. Soc. 2007, 44, 623-629.

7. Qi, X.G.; Yang, L.Z.; Liu, K. Uniqueness and periodicity of meromorphic functions concerning the difference operator. Comput. Math. Appl. 2010, 60, 1739-1746.

8. Chiang, Y.M.; Feng, S.J. On the Nevanlinna characteristic of $f(z+\eta)$ and difference equations in the complex plane. Ramanujan J. 2008, 16, 105-129.

9. Halburd, R.G.; Korhonen, R.J. Nevanlinna theory for the difference operator. Ann. Acad. Sci. Fenn. Math. 2006, 31, 463-478.

10. Hayman, W.K. Meromorphic Functions; Clarendon Press: Oxford, UK, 1964.

11. Heittokangas, J.; Korhonen, R.; Laine, I.; Rieppo, J. Uniqueness of meromorphic functions sharing values with their shifts. Complex Var. Elliptic Equ. 2011, 56, 81-92.

12. Rahman, U.; Li, X.M.; Faizullah, F. On periodicity and uniqueness of meromorphic functions. J. Comput. Theor. Nanosci. 2017, 14, 910-917.

13. Yang, C.C. On deficiencies of differential polynomials. Math. Z. 1970, 116, 197-204.

14. Zhang, J.L. Value distribution and shared sets of differences of meromorphic functions. J. Math. Anal. Appl. 2010, 367, 401-408.

15. Lahiri, I. Weighted sharing of three values and uniqueness of meromorphic functions. Kodai. Math. J. 2001, 24, 421-435.

16. Whittaker, J.M. Interpolatory Function Theory. Cambridge University Press: Cambridge, UK, 1935.

17. Halburd, R.; Korhonen, R.; Tohge, K. Holomorphic curves with shift-invariant hyperplane preimages. Trans. Am. Math. Soc. 2014, 366, 4267-4298.

18. Yang, C.C.; Hua, X.H. Uniqueness and value sharing of meromorphic functions. Ann. Acad. Sci. Fenn. Math. 1997, 22, 395-406.

19. Li, X.M.; Wen, Z.T. Uniqueness theorems of meromorphic functions sharing three values. Complex Var. Elliptic Equ. 2011, 56, 215-233.

20. Bank, S.B.; Langley, J.K. On the oscillation of certain linear differential equation in the complex domain. Proc. Edinb. Math. Soc. 1987, 30, 455-469.

21. Titchmarsh, E.C. The Theory of Functions; Oxford University Press: Oxford, UK, 1986. 
22. Mokhon'ko, A.Z. On the Nevanlinna characteristics of some meromorphic functions: Theory of Functions, Functional Analysis and Their Applications. Izd-vo Khar'kovsk Un-ta Kharkov 1971, 14, 83-87.

23. Laine, I. Nevanlinna Theory and Complex Differential Equations; Walter de Gruyter: Berlin, Germany; New York, NY, USA, 1993.

(C) 2017 by the authors. Licensee MDPI, Basel, Switzerland. This article is an open access article distributed under the terms and conditions of the Creative Commons Attribution (CC BY) license (http://creativecommons.org/licenses/by/4.0/). 